\title{
Utilizando análise semântica para descobrir implicações significantes em mapas conceituais
}

\author{
Ramon B. Moreira, Rodrigo R. Boguski, Davidson Cury ${ }^{1}$ \\ ${ }^{1}$ Departamento de Informática - Universidade Federal do Espírito Santo (UFES) \\ Vitória - ES - Brazil \\ \{rbambinil, rodrigoboguski, dedecury\}@gmail.com
}

\begin{abstract}
This paper presents a framework capable of performing semantic analysis of concept maps, through the significant implications defined by Piaget (local, systemic and structural). The objective is to provide a deeper semantic dimension in analyzing the significant implications and extract more accurate information about an individual's knowledge representation and understanding from their map representation. The framework uses natural language processing techniques to predict and word embeddings from the calculation of semantic similarity using neural networks. In order to validate its effectiveness, we carried out experiments in the classroom, obtaining very satisfactory results.
\end{abstract}

Resumo. Este artigo apresenta um arcabouço capaz de efetuar análise semântica de mapas conceituais, por intermédio das implicações significantes definidas por Piaget (local, sistêmica e estrutural). O objetivo é fornecer uma dimensão semântica mais profunda na análise das implicações significantes e extrair informações mais precisas sobre a representação do conhecimento e a compreensão de um indivíduo a partir da representação de seu mapa. O arcabouço utiliza técnicas de processamento de linguagem natural para previsão e casamento de palavras a partir do cálculo de similaridade semântica utilizando redes neurais. A fim de validar sua eficácia, realizamos experimentos em sala de aula, obtendo resultados bastante satisfatórios.

\section{Introdução}

Mapas conceituais são ferramentas gráficas utilizadas para a organização e representação do conhecimento. Eles foram desenvolvidos, em um programa de pesquisa feito por Joseph Novak no ano de 1972, em que ele procurou acompanhar e entender as mudanças na maneira como as crianças compreendiam a ciência [Novak and Musonda 1991].

Como ferramenta de apoio, os mapas conceituais podem ser utilizados em sala de aula como forma de mediar o ensino-aprendizagem, estimulando a aprendizagem significativa [Moreira 2012]. Eles são constituídos de conceitos e relações. Quando se atribui uma relação, expressa por uma frase de ligação entre dois conceitos, que contenha valor semântico, forma-se uma implicação significante.

Para Piaget [Piaget and García 1987], desde os níveis mais elementares de pensamento há implicações entre significações. Ele afirma que as implicações significantes evoluem seguindo três níveis: implicações locais nos níveis mais 
elementares, implicações sistêmicas em níveis intermediários e implicações estruturais, como os níveis mais elevados.

Este trabalho se baseia em estudos anteriormente realizados por [Moreira et al. 2019], [Rios et al. 2017] e [Dutra et al. 2006], apresentando como progresso a inserção de análise semântica na avaliação de mapas conceituais. Todas as pesquisas anteriores até então fundamentavam-se apenas nas análises sintáticas e/ou topológicas do mapa conceitual, sem considerar a significação ou sentidos das relações expressas entre conceitos. Este estudo resultou em um arcabouço para realizar a análise semântica sobre mapas conceituais a partir da observação e qualificação de implicações significantes [Piaget and García 1987] contidas nas relações nelas embarcado. A análise semântica, como sempre, objetiva uma percepção mais profunda do suposto conhecimento representado no mapa, buscando transformar indícios em evidências da aprendizagem, de fato, realizada pelo aprendiz, auxiliando o professor na avaliação dos mapas conceituais.

Este artigo está disposto da seguinte forma: a Seção 2 apresenta os mapas conceituais; a Seção 3 descreve as implicações significantes de Piaget e sua aplicação em mapas conceituais; a Seção 4 trata da análise semântica das implicações significantes no contexto de mapas conceituais e sua utilização em ambiente de sala de aula; a Seção 5 discute a análise e resultados obtidos até o momento; e finalmente, a Seção 6 apresenta as considerações finais.

\section{Mapas conceituais}

O mapeamento conceitual é uma técnica muito flexível e em razão disso pode ser usado em diversas situações, para diferentes finalidades: instrumento de análise do currículo, técnica didática, recurso de aprendizagem e meio de avaliação [Souza and Boruchovitch 2010].

De modo geral, os mapas conceituais são compostos por conceitos, que aparecem em caixas, e relações entre conceitos, que são designadas por linhas que os interligam. As palavras sobre essas linhas especificam os relacionamentos entre dois conceitos.

Proposições são enunciações sobre algum objeto ou evento no universo, seja ele natural ou artificial. Elas contêm dois ou mais conceitos conectados por palavras de ligação ou frases para compor uma afirmação com sentido. Por vezes, são chamadas de unidades semânticas ou unidades de sentido [Novak and Cañas 2006]. Por isso, mapas conceituais são um instrumento eficiente para revelar sentidos atribuídos a conceitos e relações entre os conceitos na conjuntura de um corpo de conhecimentos, de uma disciplina, de um conteúdo de sala de aula. Por exemplo, se a pessoa que fez um mapa, seja ele professor ou aprendiz, associa dois conceitos, por meio de uma ligação, então deve ser capaz de dizer o significado que observa nessa relação entre esses conceitos. A Figura 1 apresenta um exemplo de mapa conceitual.

Como instrumento de avaliação da aprendizagem, mapas conceituais podem ser usados para se obter uma visualização da organização conceitual que o aprendiz atribui a um dado conhecimento. Trata-se basicamente de uma técnica não tradicional de avaliação que busca informações sobre os significados e relações significativas entre conceitos-chave de um domínio segundo o ponto de vista do aluno. É mais apropriada para uma avaliação qualitativa, formativa, da aprendizagem [Moreira 2012]. 


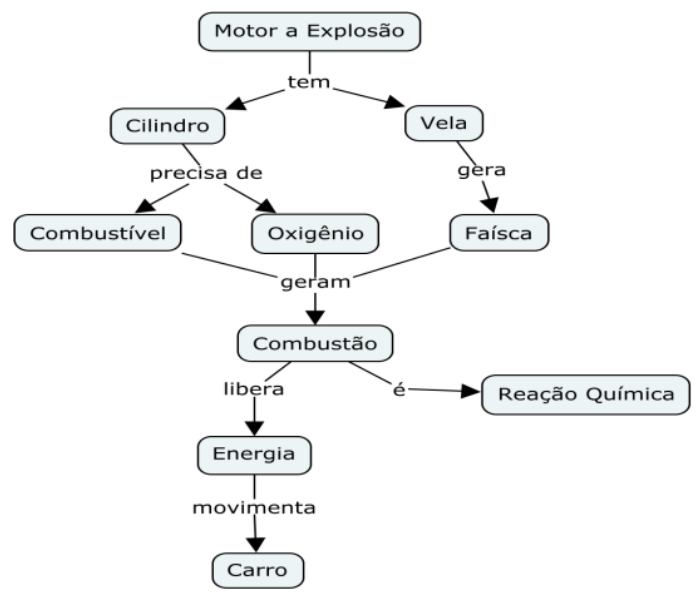

Figura 1. Exemplo de um Mapa Conceitual

\section{Implicações significantes de Piaget}

A aprendizagem pode suceder-se de diversas formas: olhando, tocando, sentindo ou ouvindo um objeto. Ao depararmos com algo novo, buscamos saber de suas propriedades, desejamos conhecer e entender suas funções e operações, assim como do que ele é capaz de fazer. Posteriormente, procuramos entender como essas operações ocorrem ou como aplicá-las de maneira diferente, e investigar do que ele é composto. Por último, descobrimos possíveis interações capazes de serem feitas com outros objetos. E assim, à medida que o conhecimento se forma, os diferentes níveis de complexidade do conhecimento vão se criando.

Piaget [Piaget and García 1987] classificou esses níveis de conhecimento e ao conjunto deles denominou implicações significantes. Elas nos permitem identificar o grau de conhecimento de uma pessoa, num dado momento e sobre um dado domínio.

Uma sentença tipo implicação transitiva $\mathrm{A} \rightarrow \mathrm{B} \rightarrow \mathrm{C}$, na abordagem de Piaget, significa que se pelo menos uma significação de $C$ está contida em qualquer uma de $B$ está, por conseguinte, também contida em qualquer de A. Exemplificando, no mapa da Figura 1, quando é dito que o motor possui vela, que por sua vez gera faísca, pela logica piagetiana, concluímos que o conceito faísca possui uma relação de significação com o conceito motor. A significação é tudo o que pode ser dito de um objeto, como uma descrição das suas propriedades, bem como tudo o que podemos observar nele. Além disso, uma implicação também é tudo o que podemos pensar dos objetos (classificá-los, estabelecer algum tipo de relacionamento etc.) [Rios et al. 2015].

Quando um indivíduo escolhe uma relação entre dois conceitos (expressa por uma frase de ligação) durante a construção de um mapa conceitual, ele está formando, em última análise, uma implicação significante. Com um conjunto de implicações, podemos identificar o grau de conhecimento de um indivíduo em um determinado domínio. A seguir, descrevemos como os três níveis das implicações podem ser representadas em mapas conceituais.

- Implicação Local - pode ser definida quando o conhecimento não ultrapassa as propriedades observáveis do objeto em um determinado contexto. Uma implicação local somente caracteriza um objeto. Elas podem qualificar um conceito ou estabelecer relações de parte do todo. Em um mapa conceitual, as implicações locais geralmente aparecem nas proposições com frases de ligação 
que usam verbos tais como é, tem, contém, possui etc., ou em predicados que qualificam o conceito.

- Implicação Sistêmica - generalizações e propriedades não diretamente observáveis começam a aparecer neste nível. A implicação sistêmica evidencia características do objeto que são notadas ou resultantes de alguma ação sobre ele. Esta implicação manifesta as relações de causa e efeito sem, contudo, revelar o por que isso ocorre. Estas relações ocorrem, em geral, pelo estabelecimento de uma relação do objeto em estudo com algum outro objeto. Para essa implicação, geralmente a causa e o efeito são, respectivamente, o sujeito e o objeto direto do verbo ou com o objeto indireto. São exemplos de verbos nocionais, que exercem ação ou evocam relação causal tais como causar, criar, fazer, gerar, disparar, produzir, emitir, resultar e assim por diante.

- Implicação Estrutural - amplia a implicação sistêmica, pois contém as razões e explicações da relação causa-efeito. Piaget fala da compreensão das razões endógenas e da descoberta das relações necessárias para o efeito ocorrer. Assim, mais do que o conhecimento de causas e efeitos, as implicações estruturais estabelecem condições que são essenciais para certas declarações, distinguindoas daquelas que são apenas suficientes. A soma das ações de vários objetos relacionados à ocorrência de mais uma ação, estabelece a existência de uma implicação desse novo nível [Rios et al. 2015]. Nos mapas, elas são identificadas a partir de relações semânticas válidas entre duas ou mais proposições que convergem para formação de um novo conceito.

Em [Moreira et al. 2019], desenvolvemos uma técnica que identificasse as implicações significantes sobre mapas conceituais e as representasse numa ferramenta de apoio a aprendizagem. As implicações são identificadas por meio de cores nas setas das proposições, para que possam ser visualizadas, sendo amarelo para implicações locais, verde para sistêmicas e azul para estruturais. Eventuais proposições que não forem classificadas por nenhuma das implicações aparecem por padrão na cor preto.

A Figura 2 demonstra o mapa conceitual da Figura 1 representado com as implicações significantes de Piaget identificadas pelo arcabouço, em que as relações em amarelo, verde e azul evidenciam, respectivamente, implicações locais, sistêmicas e estruturais. Nesse exemplo, quando dizemos que o motor tem cilindro e que a combustão é uma reação química, estamos fazendo implicações locais, já que isso pode ser registrado do objeto apenas a partir da observação de seu contexto e de seus atributos.

Quando dizemos que a energia química, liberada da reação química da combustão, é transformada em energia mecânica, que, por sua vez, faz as rodas do carro movimentarem-se, estamos denotando que são implicações sistêmicas. Para chegarmos a uma implicação estrutural precisaríamos explicar, por exemplo, que para que ocorra a combustão que ocorre dentro do cilindro do veículo, é necessário a atuação conjunta de combustível, ar e faísca. 


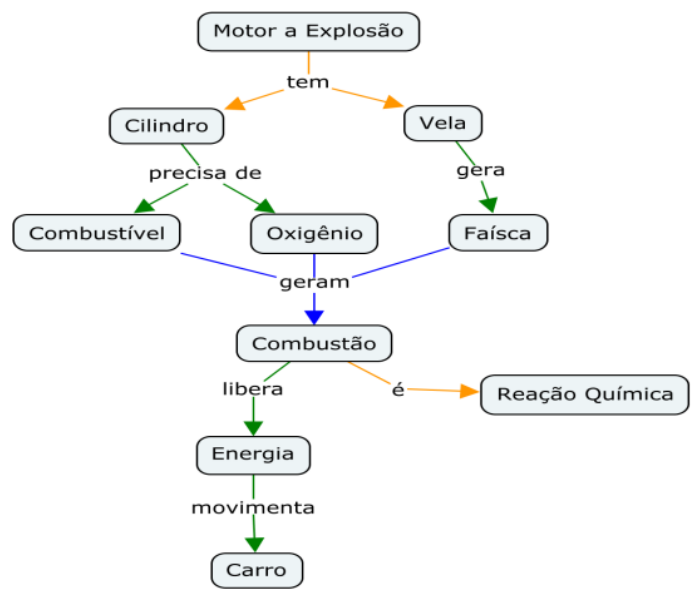

Figura 2. Mapa conceitual representando as implicações significantes

\section{Análise semântica em mapas conceituais}

A análise semântica de um mapa conceitual representa um papel importante na tradução do conhecimento humano na forma de mapas conceituais em representações rigorosas e inequívocas para processamento posterior por computadores [Hao et al. 2010]. A partir da análise semântica, é possível extrair informações mais precisas sobre a representação do conhecimento e a compreensão de um indivíduo acerca do que ele representou ou sobre o que ele sabia no momento da construção do mapa, a partir da análise significativa das proposições construídas por ele.

O arcabouço proposto é estruturado a fim de analisar, de modo progressivo, as implicações representadas em cada mapa conceitual. A dimensão semântica é obtida com a utilização de técnicas de processamento de linguagem natural (PLN) de previsão e casamento de palavras em uma frase. O modelo conceitual do arcabouço é apresentado na figura a seguir.

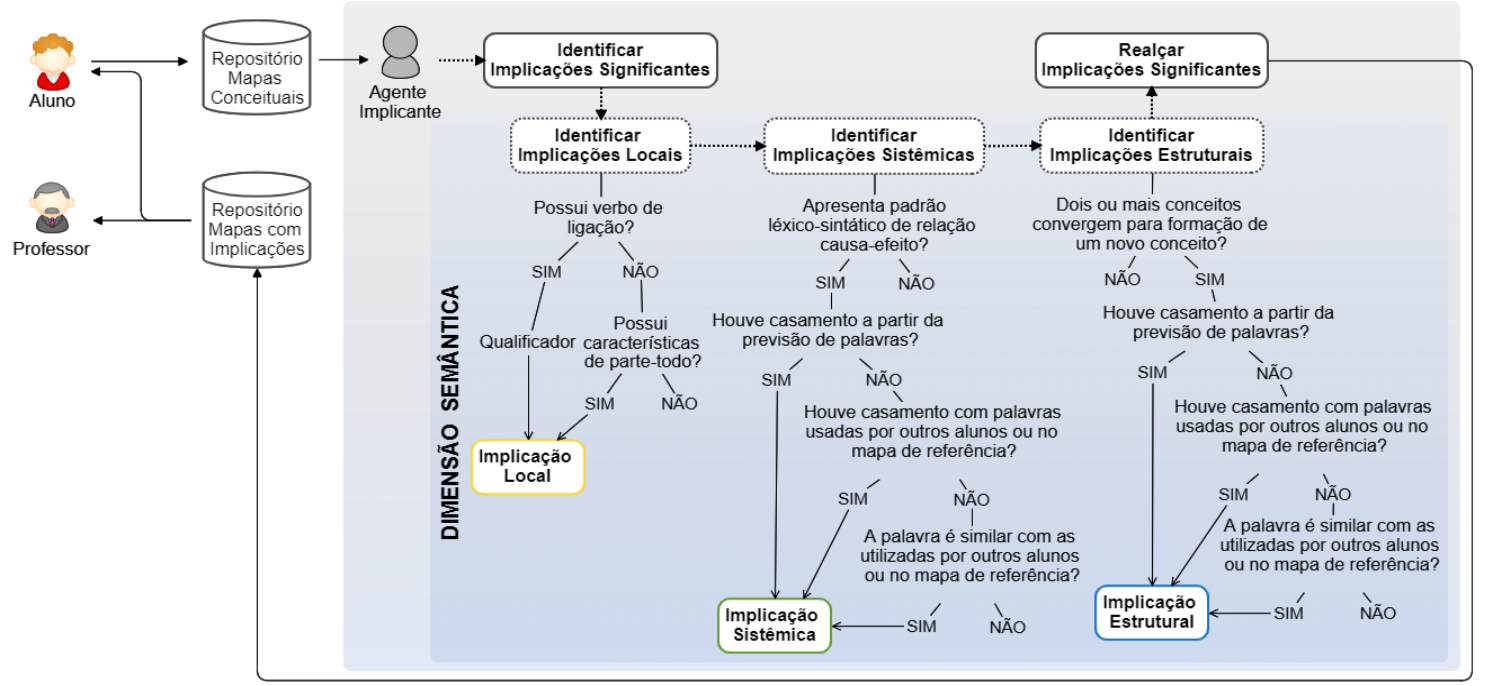

Figura 3. Modelo conceitual

O modelo é composto por dois atores, aluno e professor, e dois componentes, repositório de mapas e repositório de mapas com implicações. Os estudantes poderão acessar o arcabouço a qualquer instante para saber como está a qualidade de seu mapa. Por exemplo, se somente amarelo, mostrando apenas implicações locais, isto indicará ao 
aluno a necessidade de prosseguir até um nível superior, chegando às implicações estruturais. Já o professor, ao observar os mapas coloridos com as implicações, pode perceber em que nível um aprendiz e sua turma alcançaram em determinado momento.

O repositório de mapas tem a função de armazenar os mapas conceituais que serão construídos pelos alunos e o repositório de mapas com implicações armazena aqueles que foram analisados. Uma vez que o repositório de mapas esteja preenchido, um agente implicante busca cada mapa no repositório e analisa sua estrutura de acordo com o arcabouço desenvolvido. Ao receber um mapa conceitual, o agente implicante busca identificar as implicações significantes de Piaget, procurando primeiro por implicações locais, depois implicações sistêmicas e por último implicações estruturais.

\subsection{Implicações locais}

Para identificar implicações locais, procuramos verificar proposições cujo segundo conceito qualifica o primeiro. Neste contexto, quando um aluno utiliza um verbo de ligação, tal como ser/estar, parecer e tornar-se, a proposição passa a conter um predicativo do sujeito, o qual possui função de atribuir qualidades ao sujeito. Em relações que contenham tais verbos, é possível dizer que, semanticamente, o aluno entende que um conceito é um qualificador de outro, de forma a dar novas características a este. Caso não haja um verbo de ligação na relação, utilizamos uma segunda abordagem. Nestes casos, analisamos se o aluno está querendo representar características de parte-todo. Para isso, verificamos se há presença de verbos como ter, pegar, poder, conter, possuir. Esses verbos também caracterizam as implicações locais, porém indicam outro tipo de relação semântica, palavras que designam uma parte de um todo.

\subsection{Implicações sistêmicas}

No domínio das implicações sistêmicas, primeiro é utilizado um conjunto de padrões léxico-sintáticos definidos por [Sorgente et al. 2013] que representam a estrutura de relações causais em sentenças, seguido por uma validação semântica. Os padrões sintáticos a serem identificados são:

- Os verbos causais simples são verbos únicos que têm o significado de "ação causal" (por exemplo, gerar, disparar, criar e assim por diante);

- Os verbos frasais são frases que consistem em um verbo seguido por uma partícula (por exemplo: resulta em);

- Substantivos + preposição são expressões compostas por um substantivo seguido por uma preposição (por exemplo: causa de);

- Os verbos causativos passivos são verbos em voz passiva seguidos da preposição por (por exemplo: causado por, acionado por e assim por diante);

- Preposições simples são preposições que podem ser usadas para ligar "causa" e "efeito" (por exemplo: de, depois e assim por diante).

\subsection{Implicações estruturais}

Em relação a implicações estruturais, primeiro é feito uma vistoria na topologia do mapa conceitual, buscando ocorrências de proposições em que vários momentos conceituais convergem para formação de um outro conceito, ou seja, a junção de diferentes conceitos que implicam na formação de um novo por meio de um mesmo verbo ou locução verbal. 
Por exemplo, quando se observa, a partir da topologia do mapa conceitual da Figura 1, que os conceitos "Combustível", "Oxigênio" e "Faísca" concorrem para a construção do conceito "Combustão", significa que tais proposições são possíveis implicações estruturais e precisam passar por uma validação semântica.

\subsection{Análise semântica de implicações}

Para o nível de implicações sistêmicas e estruturais, precisamos saber se a proposição possui um valor semântico apropriado. Essa validação é feita a partir das técnicas de predição e casamento de palavras por meio da utilização de técnicas de PLN.

O primeiro passo consiste em criar uma sentença contendo os conceitos de origem com o verbo ou locução verbal da relação que os une, com o intuito de analisar sua semântica. Depois disso, é feita uma previsão de possíveis palavras que completam esta sentença naquele contexto semântico, buscando fazer um casamento de palavras.

Retomando o exemplo utilizado na subseção 4.3, temos a seguinte sentença:

\section{“Combustível, Oxigênio e Faísca geram Combustão” [1]}

A previsão de palavras é uma tarefa de processamento de linguagem natural que fornece uma frase parcial, onde as palavras mascaradas são identificadas para completar a frase a fim de produzir uma sentença que possua significado. Assim, o conceito derivado da proposição é mascarado (oculto) para que possam ser previstas e encontradas palavras que deem uma dimensão semântica à sentença.

No exemplo acima, o conceito "Combustão" é retirado da sentença e substituída pelo termo [MASK] para que se investigue se ele está contido no rol de palavras possíveis para o contexto dessa sentença. Caso ele seja encontrado, diz-se que houve um casamento de palavras, e assim estaremos diante de uma proposição com significação válida, e por conseguinte, uma implicação estrutural.

\section{"Combustivel, Oxigênio e Faísca geram [MASK]"}

Ao analisar o contexto da frase, o algoritmo apresenta, por exemplo, 100 possíveis palavras cujos sentidos são possíveis de serem utilizados naquele campo semântico. Para o exemplo da proposição formulada acima, essas são algumas das palavras retornadas que se enquadram na posição do termo [MASK]:

\section{['combustão', 'incêndios', 'reações', 'aquecimento', 'fogo', 'danos', 'acidentes', ...]}

Como pôde ser observado, a palavra "combustão" faz parte do rol de palavras que pertencem semanticamente ao contexto da proposição criada pelo autor do mapa conceitual da Figura 1. Logo, essa proposição é identificada como implicação estrutural, conforme ilustra a Figura 2.

Essa verificação de semântica, por meio de previsão de palavras, é realizada em dois níveis. No primeiro nível, realizamos uma verificação por meio de um modelo de redes neurais pré-treinado com bilhões de textos contidos em livros e na Wikipédia de forma a permitir o reconhecimento de amarrações semânticas. Caso haja uma correspondência entre a palavra do conceito derivado da relação com alguma das palavras previstas, valida-se a implicação sistêmica ou estrutural.

A previsão de palavras é realizada por um algoritmo chamado BERT (Bidirectional Encoder Representations from Transformers). É um modelo de 
representação de linguagem proposto por pesquisadores do Google AI Language que consiste em um codificador Transformer multicamada bidirecional [Devlin et al. 2018]. Ao contrário do modelo de linguagem tradicional, que prevê uma palavra a partir de seu contexto esquerdo, ou seja, a partir de palavras anteriores a ela, esse modelo prevê uma palavra a partir de seu contexto esquerdo e direito. Isso significa que ele também prevê palavras considerando vocábulos escritos à direita da palavra a ser prevista. Resultados mostraram [Devlin et al. 2018] que um modelo de linguagem treinado bidirecionalmente pode ter um senso mais profundo de contexto e fluxo de linguagem do que modelos de linguagem de direção única. Para o corpus de pré-treinamento do BERT foram utilizados o BooksCorpus ( 800 milhões de palavras) e a Wikipédia em inglês (2.500 milhões de palavras). Esse tipo de modelo consegue prever com precisão relações contextuais entre palavras.

No segundo nível, comparamos se a palavra predita se encontra também no mapa referência e nos mapas conceituais dos outros alunos. Essa abordagem, objetiva evidenciar um fechamento contextual, ampliando, dessa forma, a dimensão semântica, uma vez que, se o conceito a ser predito também aparecer nos outros mapas com um suporte e confiança mínimos, isso indicará que ele é compreensível por toda a turma de alunos, conjuntamente [Boguski \& Cury 2018]. Neste cenário, caso haja um casamento de palavras, por consequência, haverá uma implicação sistêmica ou estrutural.

Caso o conceito também exista no mapa de referência do professor, então esta implicação recebe uma pontuação com peso três. Se o conceito predito pelo modelo de previsão de palavras existir em pelo menos um mapa conceitual de outro aluno da turma, então essa implicação receberá uma pontuação com peso dois. Em caso de nenhuma ocorrência tanto nos mapas conceituais da turma quanto do professor, a implicação com o conceito predito recebe peso um. Além disso, se o conceito estiver em todos os mapas conceituais da turma, mas não estiver no mapa de referência elaborado pelo professor, pode significar alguma divergência e o professor poderá verificar o porquê de a turma ter considerado tal conceito.

Contudo, em alguns casos, a palavra buscada não possui correspondência satisfatória em nenhuma das situações anteriores. Se no exemplo da sentença 1, determinado aluno utilizasse as palavras "Calor", "Explosão" ou "Energia" ao invés do conceito "Combustão", ainda sim seriam implicações estruturais válidas semanticamente, já que essas palavras possuem uma similaridade com a palavra "Combustão". Nessa circunstância, realizamos ainda uma verificação de correspondência semântica entre palavras, como uma tentativa de encontrar similaridade de sentido entre palavras diferentes.

Para isto, utilizamos um modelo baseado em Continuous Bag of Words (CBOW): o Word2Vec ("Word to Vector"). Ele permite encontrar a similaridade semântica entre duas palavras, mesmo que sejam diferentes. Esse modelo de processamento de linguagem natural transforma palavras em vetores dimensionais de características a fim de serem comparadas, dessa forma, diferentes sentidos da palavra são combinados em um único vetor. O modelo Word2Vec gera correspondências a partir da comparação de diferentes características, possibilitando uma abordagem de grande eficácia para verificação de similaridade semântica [Mikolov et al. 2013].

Essa estratégia procura avaliar a similaridade semântica entre a palavra que o aluno utilizou e demais palavras contidas nos mapas do repositório de mapas com 
implicações. Dessa forma, o Word2Vec foi aplicado no segundo nível de análise das implicações sistêmicas e estruturais, já que ele utiliza apenas duas palavras, que serão comparadas, para encontrar a similaridade entre elas. Já o modelo BERT, citado anteriormente, gera correspondências que nos permitem ter várias representações vetoriais para a mesma palavra, com base no contexto e sentido em que ela foi usada, necessitando de uma sentença ou frase para obter melhores resultados.

Neste nível é feito uma representação vetorial para cada palavra, e quanto maior for o valor entre duas palavras, mais similares elas são. Se as palavras tiverem um alto nível de similaridade, acima de $70 \%$, significa que aquela proposição é válida e também representa uma implicação sistêmica ou estrutural.

Após processar o mapa conceitual, a última etapa dentro do agente implicante é realçar as implicações significantes, atribuindo a cor amarela para implicações locais, verde para sistêmicas e azul para estruturais.

As técnicas de PLN foram empregadas através do spaCy [Honnibal and Montani 2017], uma biblioteca de código aberto gratuita para processamento de linguagem natural avançado em Python. Ela pode ser usada para construir sistemas de extração de informações ou de compreensão de linguagem natural, ou para pré-processar texto para aprendizado profundo.

\section{Aplicação e resultados}

A fim de efetuar uma análise do arcabouço proposto, realizamos um experimento para validar a eficácia e qualidade do arcabouço, comparando seu resultado com mapas examinados por um perito humano. Essa análise foi aplicada no contexto educacional, a partir de um repositório de mapas conceituais de uma turma de alunos da disciplina de Organização e Representação do Conhecimento do curso de Arquivologia de nossa universidade. A turma já estava habituada com mapas conceituais e seus elementos, que foram trabalhados em disciplinas anteriores. A produção dos mapas foi conduzida pelo professor no ambiente de sala de aula a fim de que os alunos elaborassem um mapa conceitual sobre o assunto de Preservação Arquivística Digital, a partir de seu próprio conhecimento, sem o auxílio de material.

Para o exame quantitativo, o objetivo é avaliar a precisão do arcabouço em identificar as implicações significantes no mapa conceitual. Para isso, foram escolhidos cinco mapas conceituais do repositório que foram analisados de forma manual (professor) e automática (arcabouço) segundo a presença de implicações significantes conforme discutido nas seções 3 e 4 . A Tabela 1 apresenta os resultados da análise manual e automática para o experimento.

Tabela 1. Análise sumarizada do experimento

\begin{tabular}{|l|c|c|c|c|c|c|c|}
\hline \multirow{2}{*}{ Aluno } & \multirow{2}{*}{ Proposição } & \multicolumn{3}{|c|}{ Análise do Professor } & \multicolumn{3}{c|}{ Análise do Arcabouco } \\
\cline { 3 - 8 } & & $\begin{array}{l}\text { Implicação } \\
\text { Local }\end{array}$ & $\begin{array}{c}\text { Implicação } \\
\text { Sistêmica }\end{array}$ & $\begin{array}{c}\text { Implicação } \\
\text { Estrutural }\end{array}$ & $\begin{array}{c}\text { Implicação } \\
\text { Local }\end{array}$ & $\begin{array}{c}\text { Implicação } \\
\text { Sistêmica }\end{array}$ & $\begin{array}{c}\text { Implicação } \\
\text { Estrutural }\end{array}$ \\
\hline Aluno 1 & 12 & 2 & 8 & 2 & 1 & 8 & 2 \\
\hline Aluno 2 & 13 & 2 & 3 & 8 & 2 & 3 & 8 \\
\hline Aluno 3 & 12 & 2 & 2 & 8 & 2 & 2 & 7 \\
\hline Aluno 4 & 17 & 3 & 12 & 2 & 2 & 11 & 0 \\
\hline Aluno 5 & 16 & 0 & 4 & 10 & 0 & 3 & 10 \\
\hline TOTAL & $\mathbf{7 0}$ & $\mathbf{9}$ & $\mathbf{2 9}$ & $\mathbf{3 0}$ & $\mathbf{7}$ & $\mathbf{2 7}$ & $\mathbf{2 7}$ \\
\hline
\end{tabular}


A partir dos resultados apresentados na Tabela 1, podemos observar que $o$ arcabouço foi capaz de identificar os três tipos de implicações significantes (local, sistêmica e estrutural). Além disso, verificamos que se atingiu a eficácia de $77,77 \%$ na identificação das implicações locais, 93,10\% nas sistêmicas, e 90\% nas implicações estruturais. Ou seja, a maior parte das implicações identificadas pelo professor, também foram identificadas pelo arcabouço. Essa eficácia é calculada comparando o total de implicações identificadas manualmente pelo professor com o total identificado pelo arcabouço.

\section{Considerações finais}

O que apresentamos neste artigo é a aplicação da análise semântica em mapas conceituais por intermédio das implicações significantes de Piaget, visando identificar o conhecimento representado pelo aluno e evidenciar possíveis insuficiências na sua aprendizagem, sendo uma proposta de um novo modo de avaliação do processo de aprendizagem.

Segundo os resultados apresentados, verificamos que o arcabouço é capaz de aplicar semântica na análise de implicações significantes de Piaget progredindo estudos anteriores, já apresentando bons resultados. Esse é um estudo inicial sobre a análise semântica aplicada em mapas conceituais, havendo ainda muitos avanços que podem advir deste trabalho com o objetivo de contribuir para o seu aprimoramento e possibilitar uma melhor análise semântica dos mapas conceituais.

Como trabalhos futuros, os próximos passos são aprimorar a eficácia do arcabouço, fazendo validações semânticas mais amplas e promover a utilização de bases ontológicas para aprimorar a identificação de relações parte-todo no âmbito das implicações locais. Propomos também, integrar o arcabouço, funcionando como uma API (Application Programming Interface), em um ambiente de aprendizagem assistida por tutores inteligentes sobre mapas conceituais [Boguski et al. 2019], a fim de que a identificação automática das implicações em um mapa conceitual produzido por um aluno possa permitir que um tutor interaja com ele, em tempo real, com base no conhecimento que ele foi capaz de expor naquele instante, para obter maiores indícios de aprendizado, além da confirmação das implicações obtidas. Como consequência, o tutor pode sugerir novas ideias, novos conceitos, novos relacionamentos e levantar questionamentos ao aluno a partir daquilo que ele está representando no momento da construção do seu mapa.

As implicações significantes nos recursos de aprendizagem têm relevância para o desenvolvimento da educação. Além disso, o emprego de técnicas de processamento de linguagem natural e de análise semântica têm potencial para aperfeiçoar o aprendizado em sala de aula. Ademais, o professor ao observar um mapa colorido pelas implicações significantes, pode perceber em que nível os estudantes de sua turma alcançaram em um dado instante, resultando ainda em uma satisfatória economia cognitiva do professor ao dispensá-lo de realizar esse trabalho de forma manual.

Finalmente, esse arcabouço não pretende resolver na totalidade o problema análise semântica na identificação das implicações, porém apresenta grande potencial para auxiliar na resolução do problema cuja solução depende de outras intervenções. 


\section{Referências}

Boguski, R. R., \& Cury, D. (2018). Usando regras de associação para a identificação de falhas conceituais. Simpósio Brasileiro de Informática na Educação (SBIE), (pp. 1443-1453). Fortaleza.

Boguski, R. R., Cury, D., and Gava, T. (2019). Tom: An intelligent tutor for the construction of knowledge represented in concept maps. In 2019 IEEE Frontiers in Education Conference (FIE), pages 1-7. IEEE.

Devlin, J., Chang, M.-W., Lee, K., and Toutanova, K. (2018). Bert: Pre-training of deep bidirectional transformers for language understanding. arXiv preprint arXiv:1810.04805.

Dutra, Í. M., Johann, S. P., Piccinini, C. A., and da Cruz Fagundes, L. (2006). Uma base de dados para compartilhamento de experiências no uso de mapas conceituais no acompanhamento de processos de conceituação. Novas Tecnologias na Educação, CINTED-UFRGS.

Hao, J.-X., Yan, A., and Chi-Wai, R. (2010). A semantic analysis method for concept map-based knowledge modeling. In Proceedings of the 1st International Conference on E-Business Intelligence (ICEBI2010), Atlantis Press.

Honnibal, M. and Montani, I. (2017). spaCy 2: Natural language understanding with Bloom embeddings, convolutional neural networks and incremental parsing. To appear, v. 7, n. 1, p. 411-420, 2017.

Mikolov, T., Sutskever, I., Chen, K., Corrado, G. S., and Dean, J. (2013). Distributedrepresentations of words and phrases and their compositionality. InAdvances in NeuralInformation Processing Systems, pages 3111-3119.

Moreira, M. A. (2012). Mapas conceituais e aprendizagem significativa (concept maps and meaningful learning). Aprendizagem significativa, organizadores prévios, mapas conceituais, digramas $V$ e Unidades de ensino potencialmente significativas, page 41.

Moreira, R., Aguiar, C., and Cury, D. (2019). Ferramenta computacional multilíngue para analisar significações em mapas conceituais. In Brazilian Symposium on Computers in Education (Simpósio Brasileiro de Informática na Educação-SBIE), volume 30, page 419.

Novak, J. D. and Cañas, A. J. (2006). The theory underlying concept maps and how to construct and use them. research report 2006-01 Rev 2008-01, Florida Institute for Human and Machine Cognition.

Novak, J. D. and Musonda, D. (1991). A twelve-year longitudinal study of science concept learning. American educational research journal, 28(1):117-153.

Piaget, J. and García, R. (1987). Hacia una lógica de significaciones. Gedisa.

Rios, P., Cury, D., and Dutra, Í. M. (2015). Automatizando uma argumentação construtivista por meio dos mapas conceituais. In $X X$ Congreso Internacional de Informática Educativa (TISE'2015). Disponivel em: http://www.tise. cl/volumen11/TISE2015/157-162.pdf. 
Rios, P., Teodoro, G., Aguiar, C., and Cury, D. (2017). Uma abordagem construtivista para a identificar o conhecimento usando mapas conceituais. In Brazilian Symposium on Computers in Education (Simpósio Brasileiro de Informática na Educação-SBIE), volume 28, page 394 .

Sorgente, A., Vettigli, G., and Mele, F. (2013). Automatic extraction of cause-effect relations in natural language text.DART@AI*IA, 2013:37-48.

Souza, N. A. d. and Boruchovitch, E. (2010). Mapas conceituais: estratégia de ensino/aprendizagem e ferramenta avaliativa. Educação em Revista, 26:195-217. 